

\title{
Nitruration initiale par NH3 à basse température de $\operatorname{Si}(111) 1 \times 1-H$
}

\author{
J. Lacharme, N. Safta, J. Bonnet, C. Casado, M. Iqbal, C. Sebenne
}

\section{To cite this version:}

J. Lacharme, N. Safta, J. Bonnet, C. Casado, M. Iqbal, et al.. Nitruration initiale par NH3 à basse température de Si(111) 1x1-H. Journal de Physique IV Proceedings, 1994, 04 (C9), pp.C9-155-C9-158. 10.1051/jp4:1994924 . jpa-00253485

\section{HAL Id: jpa-00253485 https://hal.science/jpa-00253485}

Submitted on 1 Jan 1994

HAL is a multi-disciplinary open access archive for the deposit and dissemination of scientific research documents, whether they are published or not. The documents may come from teaching and research institutions in France or abroad, or from public or private research centers.
L'archive ouverte pluridisciplinaire HAL, est destinée au dépôt et à la diffusion de documents scientifiques de niveau recherche, publiés ou non, émanant des établissements d'enseignement et de recherche français ou étrangers, des laboratoires publics ou privés. 


\title{
Nitruration initiale par $\mathrm{NH}_{3}$ à basse température de $\mathrm{Si}(111) \mathrm{1x1}-\mathrm{H}$
}

\author{
J.P. Lacharme, N. Safta, J.E. Bonnet*, C. Casado*, M. Iqbal et C.A. Sebenne \\ Laboratoire de Physique des Solides, URA 154 du CNRS, Université P. et M. Curie, BP. 79, 75252 Paris \\ cedex 05, France \\ * LURE, associé CNRS-CEA, Bât. 209D, Université de Paris-Sud, 91405 Orsay cedex, France
}

\begin{abstract}
Silicon samples have been chemically treated in order to get a Si(111) 1x1: H surface under ultrahigh vacuum. This surface was exposed to doses of $\mathrm{NH}_{3}$ in the $10^{2}$ to $10^{3}$ Langmuir range, either kept at room temperature or Joule-heated at $400^{\circ} \mathrm{C}$. $\mathrm{The} \mathrm{Si}_{2 \mathrm{p}}$ core level spectra were recorded upon synchrotron light beam excitation at a surface - sensitive photon energy wavelength of $128 \mathrm{eV}$. The $\mathrm{N}_{2 \mathrm{~s}}$ nitrogen peak binding energy at $20 \mathrm{eV}$ was also recorded.

While at room temperature the hydrogenated surface remained unperturbed whatever the dose of unexcited $\mathrm{NH}_{3}$, a reaction occurred as soon as the temperature reached about $400^{\circ} \mathrm{C}$. The large chemical shift of the $\mathrm{Si}_{2} \mathrm{p}$ peak shows that nitrogen atoms are imbedded in the silicon surface. The temperature of $400^{\circ} \mathrm{C}$ at which the reaction occurs is below the lowest one at which $\mathrm{H}$ starts leaving the purely hydrogenated surface (over $450^{\circ}$ C). It is also lower than the temperature needed to start loosing hydrogen from a clean $\mathrm{Si}(111)$ surface which has been saturated by $\mathrm{NH}_{3}$ : these results suggest that the $\mathrm{H}$-induced $1 \mathrm{x} 1$ relaxed reconstruction of the $\mathrm{Si}(111)$ surface facilitates the surface reaction of nitridation in a similar way as alkali metal atoms enhance the oxidation process along $\mathrm{Si}$ surfaces.
\end{abstract}

\section{INTRODUCTION}

La nitruration des surfaces de silicium peut être obtenue directement à parti de la molécule $\mathrm{N}_{2}$, soit par réaction à haute température $\left(\geq 1000^{\circ} \mathrm{C}\right)$ [1] soit par ionisation [2], ou à partir de la molécule $\mathrm{NH}_{3}$ à des températures relativement basses $\left(625^{\circ} \mathrm{C}\right)[3,4]$. L'intérêt de ce dernier procédé est d'éviter la création de défauts sur la surface. Ces diverses expériences réalisées principalement sur la surface $\mathrm{Si}(100) 2 \times 1[5,6]$, plus rarement sur la face $\mathrm{Si}(111) 7 \times 7$ [7] ont montré le rôle important des liaisons pendantes dans la dissociation de la molécule $\mathrm{NH}_{3}$ puis dans la formation du composé $\mathrm{SiN}_{\mathrm{X}}$. D'autres voies permettent également de nitrurer le silicium dès $500^{\circ} \mathrm{C}$ avec $\mathrm{N}_{2}$ en utilisant un métal alcalin $\mathrm{Na}$ ou $\mathrm{K}$ qui joue alors le rôle de catalyseur dans la réaction [8], sans liaisons pendantes cette fois.

Une nitruration par $\mathrm{NH}_{3}$ peut-elle avoir lieu sur des surfaces de silicium dont toutes les liaisons pendantes ont été supprimées par hydrogénation ? C'est pour répondre à cette question que la présente étude a été menée et dont les résultats de photoémission des niveaux de coeur obtenus sur la ligne PES2 de l'anneau SUPERACO du LURE sont présentés ici.

\section{EXPERIENCE}

Les mesures ont été réalisées sur des échantillons de silicium de type $\mathrm{n}$ à $10^{16}$ atomes $\mathrm{As} / \mathrm{cm}^{3}$. Le plan de découpe (111) est défini à $0,02^{\circ}$ près. Les échantillons utilisé $(20 \times 5 \times 0.5$ $\mathrm{mm}^{3}$ ) ont subi un traitement d'oxydation thermique. L'hydrogénation de la surface $\mathrm{Si}(111)$ se fait avant la mise sous vide de l'échantillon suivant une procédure qui consiste à dissoudre tout d'abord la couche de $\mathrm{SiO}_{2}$ par une attaque fluorhydrique puis à fixer l'hydrogène par une solution $\mathrm{NH}_{4} \mathrm{~F}$ de $\mathrm{P}_{\mathrm{H}}$ approprié [9]. La surface ainsi obtenue est dite $\mathrm{Si}(111) 1 \times 1-\mathrm{H}$ et présente un plan (111) idéal d'atomes Si liés chacuns à un atome $\mathrm{H}$. 
Les mesures de photoémission sous faisceau synchrotron sont effectuées après changement de l'échantillon dans une enceinte à ultravide dont la pression limite n'excède pas 2 à $3 \times 10^{-10}$ Torr à l'aide d'un analyseur énergétique et angulaire hémisphérique d'électrons (résolution $0.35 \mathrm{eVà} 100 \mathrm{eV}$ ). Un diffractomètre d'électrons lents, une source de rayons $\mathrm{X}$ et un analyseur de gaz complètent le dispositif.

Le chauffage de l'échantillon est obtenu par effet Joule et la température mesurée directement par un pyromètre à infrarouge. La réaction a eu lieu en portant l'échantillon à $400^{\circ} \mathrm{C}$ et en présence de $\mathrm{NH}_{3}$ de haute pureté à $10^{-6}$ Torr pendant 90 secondes. L'échantillon est ramené à la température ordinaire dès la fermeture de la vanne d'introduction de gaz.

Les spectres de photoémission ont été enregistrés pour des photons de $128 \mathrm{eV}$ à $45^{\circ}$ d'incidence. L'analyse des électrons photoémis se fait suivant la normale à la surface. L'énergie cinétique des électrons analysés est comprise entre 20 et $30 \mathrm{eV}$, ce qui privilégie l'analyse de la surface de l'échantillon par rapport au volume [10] pour le niveau de coeur $\mathrm{Si}_{2} \mathrm{p}$.

Un spectre a été enregistré avec une énergie de photons de $54 \mathrm{eV}$ afin de vérifier, d'une part, la présence d'azote sur la surface (par le niveau $\mathrm{N}_{2 \mathrm{~s}}$ situé à environ $20 \mathrm{eV}$ sous le niveau de Fermi) [4] et, d'autre part, l'absence de contamination par l'oxygène dont le niveau $\mathrm{O}_{2 s}$ est situé à $25 \mathrm{eV}$ sous $\mathrm{E}_{\mathrm{F}}[11]$.

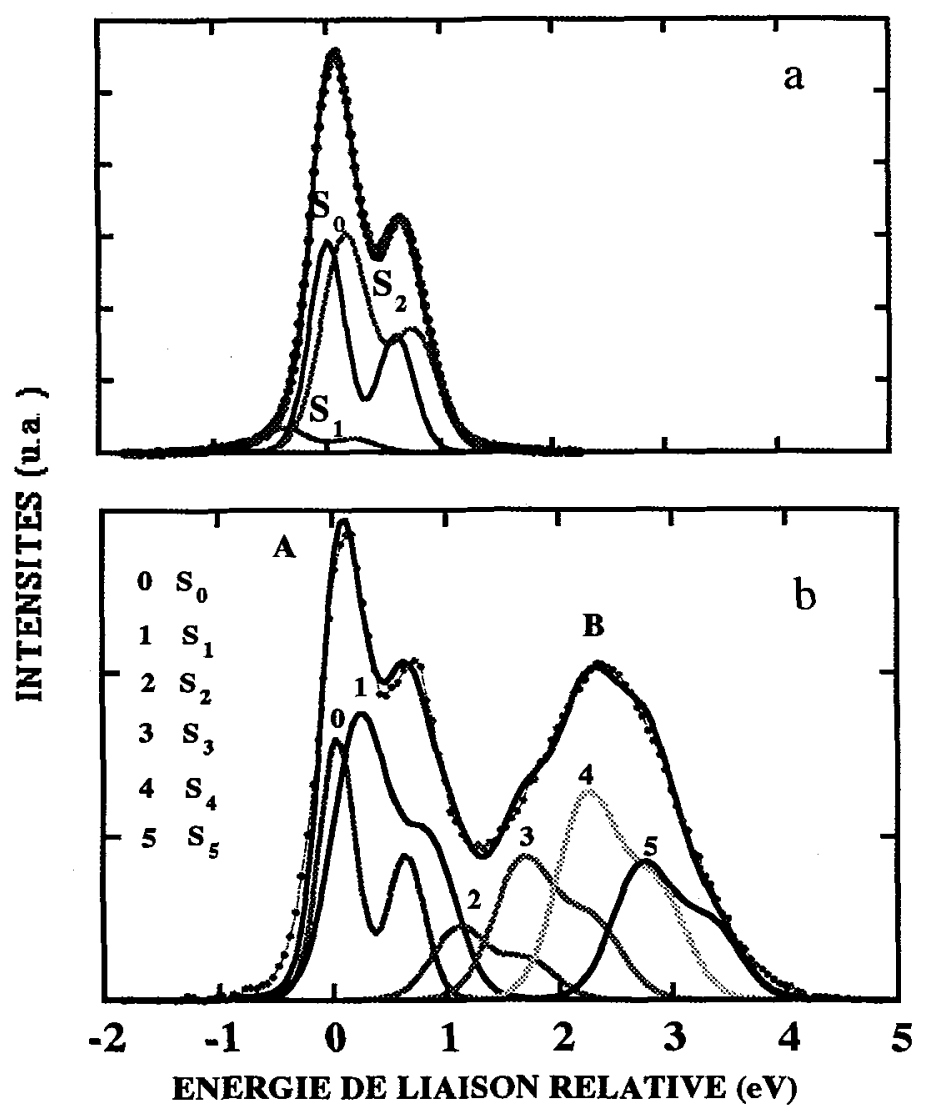

Fig. 1 : Spectres du niveau de coeur $\mathrm{Si}_{2} \mathrm{p}$ en fonction de l'énergie de liaison relative à la composante de volume, ( hv = $128 \mathrm{eV}$, incidence $45^{\circ}$ et comptage des photoélectrons émis suivant la normale à la surface).a : pour une surface (111) de silicium hydrogénée $1 \times 1, b$ : pour la même surface exposée à 90 Langmuirs de $\mathrm{NH}_{3}$ à $400^{\circ} \mathrm{C}$. 


\section{RESULTATS ET DISCUSSION}

\subsection{Surface $\operatorname{Si}(111) \quad 1 \times 1: H$}

L'absence de reconstruction de la surface après hydrogénation est vérifiée par diffraction d'électrons lents qui donne un diagramme $1 \times 1$ très fin et contrasté.

Le spectre de photoémission du niveau $\mathrm{Si}_{2}$ de la surface hydrogénée est représenté par la figure 1-a après un premier traitement des résultats consistant à éliminer le fond continu des électrons rétrodiffusés et secondaires. La disparition des états de surface est déjà visible sur le spectre brut puisqu'aucune structure n'est manifestement présente au pied du pic, du côté des énergies de liaisons plus faibles, cette composante est présente sur $\operatorname{Si}(111) 7 \times 7$ [10] comme sur $\mathrm{Si}(100) 2 \times 1$ [12] et caractérise les états de surfaces occupés correspondant à certains atomes de la surface.

La décomposition du spectre repose sur le choix délibérément simplifié d'une composante de volume $S_{0}$ et de deux composantes de surface $S_{1}$ et $S_{2}$ correspondant respectivement aux atomes de silicium de volume et aux atomes de silicium liếs aux hydrogènes (qui définissent le plan de la surface). Chaque composante est dédoublée en deux pics $2 \mathrm{p} 1 / 2$ et $2 \mathrm{p} 3 / 2$ dont le rapport des intensités $\mathrm{I}_{\mathrm{p} 1 / 2}$ et $\mathrm{I}_{\mathrm{p} 3 / 2}$ et la distance énergétique sont égales respectivement à 0.55 et $0.605 \mathrm{eV}$, valeurs conformes à celles usuellement utilisés $[10,13]$

Chaque pic est le produit de convolution d'une gaussienne de largeur g correspondant à la résolution de l'analyseur et d'une lorentzienne de largeur $0,035 \mathrm{eV}$ caractéristique de la largeur du niveau.

\subsection{Surface Si(111)-H après réaction $\mathrm{NH}_{3}$}

Le spectre de photoémission de $\mathrm{Si}_{2}$ (figure 1-b) est caractéristique de la surface après fixation d'azote dont le niveau $\mathrm{N}_{2 \mathrm{~s}}$ apparaît, par ailleurs, de façon très visible sur le spectre de la bande de valence enregistrée pour des photons de $54 \mathrm{eV}$. Aucune structure à $25 \mathrm{eV}$ sous le niveau de Fermi correspondant au niveau $\mathrm{O}_{2}$ s'apparaît dans les résultats, ce qui confirme l'absence de contamination par l'oxygène.

On distingue sur la figure 1-b en partant des énergies de liaison les plus faibles une structure $A$ constituée en partie du doublet $S_{0}$ caractéristique des atomes $\mathrm{Si}$ du volume et une structure B plus large caractéristique des liaisons $\mathrm{Si}-\mathrm{N}$.

L'intensité de B est proportionnelle à la quantité d'azote fixée sur la surface. Cette quantité peut être évaluée en comparant l'intensité de $\mathrm{B}$ à celle obtenue dans des conditions expérimentales identiques pour un recouvrement connu d'azote sur silicium [14 ]. On arrive ainsi, pour le spectre de la figure 1-b, à un recouvrement en azote compris entre 0.75 et 1.2 monocouche (une monocouche correspond à $7,810^{14}$ atomes par $\mathrm{cm}^{2}$ ).

S'il semble raisonnable d'admettre que les atomes d'azote se répartissent dans le plan de la surface (ou dans son voisinage) en s'entourant de 3 atomes de silicium, aucun indice ne permet cependant de connaître la composition exacte de cette couche de composé Si-N. En particulier, la présence d'atomes résiduels d'hydrogène est très probable en raison de la présence évidente dans la structure $A$ d'une composante $S_{1}$ de surface proche de la composante $S_{2}$ de la figure 1-a attribuée à $\mathrm{Si}-\mathrm{H}$.

La décomposition du spectre de la figure 1-b est faite suivant une procédure utilisée dans le traitement des résultats de photoémission relatifs à l'oxydation de $\mathrm{Si}(111)[15,16$ ] ou la nitruration du silicium amorphe [17]. Le spectre comporte donc un doublet de volume $S_{0}$ et cinq composantes de surfaces $S_{1} \rightarrow S_{5}$ correspondant pour $S_{2} S_{3} S_{4} S_{5}$ à des degrés de nitruration croissants des atomes de silicium.

$\mathrm{S}_{5}$ est attribuée au silicium entouré de $4 \mathrm{~N}$ composé de $\mathrm{Si}_{3} \mathrm{~N}_{4}$ auquel correspond un déplacement chimique du niveau $\mathrm{Si}_{2}$ compris entre 2.7 et $3 \mathrm{eV}[2,8]$. La position énergétique de $\mathrm{B}$ par rapport à $\mathrm{S}_{0}$ (ou $\mathrm{A}$ ) est égale à $2.25 \pm 0.05 \mathrm{eV}$, cette valeur montre que la composante $\mathrm{S}_{5}$ 'est pas la composante principale dans $\mathrm{B}$.

La décomposition représentée sur la figure 1 correspond au meilleur ajustement des résultats expérimentaux à partir de 6 composantes ; les paramètres de ces composantes sont donnés dans le tableau 1 ( en eV): 


\begin{tabular}{cccccc}
\cline { 2 - 6 } & $\begin{array}{c}\text { Position } \\
\pm 0.01 \mathrm{eV}\end{array}$ & $\begin{array}{c}\text { Partage } \\
\mathrm{S}-0\end{array}$ & $\begin{array}{c}\text { Rapport } \\
\mathrm{I}_{1 / 2} / \mathrm{I}_{3 / 2}\end{array}$ & $\begin{array}{c}\text { Largeur } \\
\text { Gaussienne }\end{array}$ & $\begin{array}{c}\text { Largeur } \\
\text { Lorentzienne }\end{array}$ \\
\hline $\mathrm{S}_{0}$ & 0 & 0,605 & 0,55 & 0,35 & 0,035 \\
\hline $\mathrm{S}_{1}$ & 0,25 & 0,605 & 0,55 & 0,40 & 0,035 \\
\hline $\mathrm{S}_{2}$ & 1,085 & 0,605 & 0,55 & 0,50 & 0,035 \\
\hline $\mathrm{S}_{3}$ & 1,69 & 0,605 & 0,55 & 0,50 & 0,035 \\
\hline $\mathrm{S}_{4}$ & 2,25 & 0,605 & 0,55 & 0,50 & 0,035 \\
\hline $\mathrm{S}_{5}$ & 2,71 & 0,605 & 0,55 & 0,55 & 0,035 \\
\hline
\end{tabular}

Le faible déplacement chimique de $S_{1}$ montre que cette structure correspond soit à la présence de $\mathrm{Si}-\mathrm{H}$ soit à des atomes de silicium dont l'environnement atomique est proche de celui des atomes $S i$ de volume. Dans ce dernier cas, la composante $S_{1}$ pourrait donc représenter les atomes Si de l'interface entre le volume et la couche superficielle qui ,elle,serait principalement constituée d'atomes Si liés à $3 \mathrm{~N}$ et $1 \mathrm{Si}$ (composante $\mathrm{S}_{4}$ ) ; $\mathrm{S}_{2} \mathrm{~S}_{3}$ et $\mathrm{S}_{5}$ correspondraient aux atomes Si d'environnements complémentaires.

En conclusion, les spectres de photoémission à haute résolution de la surface $\mathrm{Si}(111)$ $1 \times 1 / \mathrm{H}$ exposée à $\mathrm{NH}_{3}$ à une température de $400^{\circ} \mathrm{C}$ présentent un déplacement chimique du niveau $\mathrm{Si}_{2} \mathrm{p}$ dû à la formation d'un composé superficiel $\mathrm{SiN}_{\mathrm{X}}$. Le traitement des résultats fait apparaître 6 composantes correspondant pour 4 d'entre d'elles à Si entouré de 1,2,3 ou 4 atomes $\mathrm{N}$, avec une proportion plus grande d'atomes $\mathrm{Si}$ entourés de 3 atomes $\mathrm{N}$ à la surface. La quantité d'azote fixée est proche de la monocouche. L'hydrogène joue un rôle essentiel de catalyseur dans cette nitruration à partir de $\mathrm{NH}_{3}$.

\section{REMERCIEMENTS}

Nous sommes extrèmement reconnaissants au Dr. Paul DUMAS pour la qualité des échantillons de silicium hydrogéné qu'il a bien voulu nous préparer.

\section{REFERENCES}

[1] Adams A.C., VLSI Technology, edited by Sze S.M. (Mc Graw. Hill, New-York, 1983).

[2] Maillot C., Roulet H. et Dufour G., J. Vac. Sci. Technol. B 2 (3) (1984) 316.

[3] Chérif S.M., Lacharme J.P. et Sébenne C.A., Surf. Sci. 262 (1992) 33.

[4] Bischoff J.L. et al., Surf. Sci. 293 (1993) 35.

[5] Moriarty N.W. et Smith P.V., Surf. Sci. 265 (1992).

[6] Hlil E.K., Kubler L., Bischoff J.L. et Bolmont D., Phys. Rev. B 35 (1987) 5913.

[7] Bozso F. et Avouris Ph., Phys. Rev. B 38 (1988) 3937.

[8] Soukiassian P., Bakohi M.H., Starnberg H.J. et Hurych Z., J. Vac. Sci. Technol. A 6 (3) (1988) 1535.

[9] . Higashi G.S., Chabal Y.J., Trucks G.W. et Krishnan R., Appl. Phys. Lett. 56 (1990) 656.

[10] Karlsson C.J. et al., Phys. Rev. B 41 (1990) 1521.

[11] Ranke W. et.Xing Y.R., Surf. Sci. 157 (1985) 353.

[12] Rich D.H., Miller T. et Chiang T.C., Phys. Rev. B 37 (1988) 3124.

[13] Hricovini K. et al., Phys. Rev. Lett. 70 (1993) 1992.

[14] Chérif S.M., Lacharme J.P. et Sébenne C.A., Surf. Sci. 243 (1991) 113.

[15] Himpsel F.J. et al., Phys. Rev. B 38 (1988) 6084.

[16] Rochet F. et al., Appl. Surf. Sci. 59 (1992) 117.

[17] Kärcher R., Ley L. et Johnson R.L., Phys. Rev. B 30 (1984) 1896. 